Revista Científica de FAREM-Estelí

Medio ambiente. tecnología y desarrollo humano

Año 9 | Núm. 35| Julio-septiembre, 2020

ISSN: 2305-5790

https://rcientificaesteli.unan.edu.ni

DOI: https://doi.org/10.5377/farem.v0i35.10278

\title{
Actividad fisiológica, alimenticia y psicológica de los adultos mayores durante la cuarentena del Covid-19 en el Centro de Atención Integral (CEAM) la Delicia. Quito, Ecuador
}

\section{The physiological, nutritional, and psychological activity of the elderly during the quarantine of Covid-19 at the Centro de Atención Integral (CEAM) la Delicia. Quito, Ecuador}

Dalinda de los Ángeles Cepeda Ortiz ${ }^{1}$ dcepeda@unibe.edu.ec

Janeth Stefania Jácome Arboleda² sjacome@unibe.edu.ec

\section{RESUMEN}

Recibido: 24 de junio de 2020, Aceptado: 09 de septiembre de 2020

El brote epidémico SARS-CoV-2 se ha convertido en una pandemia por la enfermedad COVID19, para contener de una manera más eficaz la enfermedad es fundamental establecer un período de cuarentena óptimo para que aproximadamente el $95 \%$ de los casos sean retenidos y reducir el riesgo de transmisión. El estudio tiene como objetivo, analizar la actividad física, alimentaria y psicológica emergente del estatus QUO en los adultos mayores del CEAM la Delicia-Ecuador, en estado de cuarentena Covid19. Se aplicó una metodología mixta de diseño exploratorio secuencial, para la recolección de datos se ejecutó un análisis teórico documental y un cuestionario de opción múltiple. Los datos fueron analizados a través de la triangulación o contrastación de información. Los resultados demuestran que el sedentarismo induce a que los adultos mayores disipen sus niveles funcionales de forma acelerada. El estrés y miedo psicológico se relaciona con la estabilidad sedentaria que se establece por la cuarentena, provocando insurrecciones en el sueño, generando ansias de digerir alimentos. Los investigadores recomiendan una alimentación sana en fibras y vitaminas, y promover el ejercicio en la población de edad avanzada, ya que esta situación se considera un problema clínico y de salud pública.

Palabras clave: alimentación; actividad física; psicología; adulto mayor; COVID-19.

\begin{abstract}
The SARS-CoV-2 outbreak has become a pandemic for COVID19 disease, and to more effectively contain the disease it is essential to establish an optimal quarantine period for approximately $95 \%$ of cases to be retained and to reduce the risk of transmission. The study aims to analyze the physical, nutritional, and psychological activity emerging from the QUO status in the elderly of CEAM La Delicia - Ecuador in a Covid19 quarantine period. A mixed sequential exploratory design methodology was applied, theoretical documentary analysis, and a multiple-choice questionnaire were executed for data collection. The data were analyzed through triangulation or information contrast. The results show that sedentarism induces older adults to dissipate their functional levels in an accelerated manner. Stress and psychological fear are related to the sedentary stability that is established by the quarantine, causing insurrections in sleep, generating anxiety to digest food. Researchers recommend healthy food in fibers and vitamins and promote exercise in the elderly population since this situation is considered a clinical and public health problem.
\end{abstract}

Keywords: food; physical activity; psychology; elderly; COVID-19.

1 Universidad Iberoamericana del Ecuador UNIB.E. Quito av Colon, Ecuador. Orcid: http://orcid.org/0000-0003-3045-5762

2 Universidad Iberoamericana del Ecuador UNIB.E. Quito av Colon, Ecuador. Orcid: http://orcid.org/0000-0003-0788-7591

(C) 2020 - Revista Científica de FAREM-Estelí.

(c) (i) Este trabajo está licenciado bajo una Licencia Internacional Creative Commons 4.0 Atribución-NoComercial- 


\section{INTRODUCCIÓN}

A medida que la población mayor continúa extendiéndose la esperanza de vida aumenta. La preocupación central es si el tiempo adicional comprende años de vida saludable y promueve una alta calidad de vida relacionada con la salud hasta la vejez. Es bien sabido que el envejecimiento causa cambios graduales en el organismo, lo que conduce a la pérdida de función, debilidad, enfermedad y muerte. También de forma científica se reconoce que la actividad física permite una mejor condición fisiológica al contrario de las personas mayores que se encuentran entre el segmento más sedentario y físicamente inactivo de la sociedad (Seong et al., 2020).

Por lo anterior, se hace un análisis de la situación actual de la actividad física en el adulto mayor que acuden a los Centros de Experiencia del Adulto Mayor (CEAM), ubicado en la Delicia, Patronato Municipal de San José, ciudad Quito, Ecuador. Debido estado de cuarentena por Covid - 19, están obligados a permanecer en sus casas con distanciamiento social debido a que son un grupo vulnerable. El CEAM es un proyecto emblemático del Municipio de Quito. Cuenta con más de 17.000 usuarios y usuarias que se benefician de sus servicios gratuitos en los más de 514 puntos distribuidos estratégicamente en la provincia de Pichincha ciudad Quito.

Los objetivos del CEAM son mejorar la calidad de vida de los adultos mayores que habitan en el Distrito Metropolitano de Quito a través del proyecto 60 y Piquito, enfocado en la recreación mediante talleres de actividad física, baile, taichí, yoga, música, manualidades, gastronomía, cosmetología, salidas turísticas, excursiones, recorridos a museos, parques, entre otras. De igual forma el CEAM brinda actividades saludables a través de sesiones de fisioterapia para prevenir y controlar patologías propias de la edad. Visitas solidarias en coordinación con las Administraciones Zonales para atender a los adultos mayores en sus hogares, con profesionales en las ramas de psicología, fisioterapia y trabajo social.

El CEAM genera un apoyo académico-productivo, mediante convenios con entidades académicas para capacitar sobre negocios y emprendimientos que serán promocionados en las ferias productivas que organiza el Patronato San José.

La OMS (2015), menciona que los adultos de 65 en adelante deben dedicar 150 minutos semanales a realizar actividades físicas moderadas, la actividad se practicará en sesiones de 10 minutos, como mínimo para obtener mayores beneficios para la salud. La OMS (2015), define al envejecimiento activo como el proceso de optimizar las oportunidades de salud, participación y seguridad en orden a mejorar la calidad de vida de las personas que envejecen. De allí se deriva cual es la importancia de generar hábitos de vida saludables enfocados en la actividad física, generando un buen uso del tiempo libre y por consiguiente una vejez armoniosa.

Para Myers et al., (2020) las actividades físicas (AF) se definen como cualquier movimiento corporal producido por los músculos esqueléticos que resultan en gasto de energía. La actividad física abarca el ejercicio, los deportes y las actividades físicas realizadas como parte de la vida diaria, la ocupación, el ocio o el transporte activo. El ejercicio es una subcategoría de AF que 
está planificada, estructurada y es repetitiva y que tiene como objetivo final o intermedio el mejoramiento o el mantenimiento de la condición física. La función física es la capacidad de un individuo para realizar las actividades físicas de la vida diaria. La función física refleja la función y el control motor, la aptitud física y la AF habitual (Andrade et al., 2020).

La AF es un factor protector para las enfermedades no transmisibles, como las enfermedades cardiovasculares, los accidentes cerebrovasculares, la diabetes y algunos tipos de cáncer según (Wang y Chen, 2019). La AF se asocia con una mejor salud mental, un retraso en la aparición de la demencia y una mejor calidad de vida y bienestar. Los beneficios para la salud de la AF están bien documentados, ya que los niveles más altos y la mayor frecuencia de la AF se asocian con un menor riesgo y una mejor salud en varias áreas clave (Volkwein y McConatha, 2018).

Por otra parte, al igual que la AF la alimentación establece un factor clave en la población adulta. Una preocupación principal de muchas personas mayores y sus cuidadores es la movilidad. Las disminuciones en la movilidad física son críticas porque están fuertemente alineadas con el riesgo de discapacidad, deterioro cognitivo, institucionalización, caídas y mortalidad (Seong et al., 2020). Como paradigma bien desarrollado, un enfoque nutricional en el envejecimiento ha sido merecidamente en la salud ósea para prevenir la osteopenia y la osteoporosis (Jang, Kim y Oh 2018).

Sin embargo, a diferencia de una fractura, no existe un criterio de valoración clínico bien definido para la disminución y pérdida de la función del músculo esquelético o lo que se denomina sarcopenia. Sin embargo, como el hueso, la masa y la función del músculo esquelético son modificables de forma inmanente, de manera análoga al hueso, el músculo esquelético es mecánicamente sensible y es estimulado para girar, favoreciendo la acumulación neta, con la carga. También se ha demostrado que la proteína dietética es un factor dietético modificable que, cuando se consume en cantidades superiores a las recomendadas, es decir, la cantidad diaria recomendada de $0.8 \mathrm{~g} \cdot \mathrm{kg}$ $-1 \cdot d-1$, puede reducir las disminuciones en la masa muscular según (Shlisky et al., 2017).

Críticamente, los requerimientos de proteína en la dieta en las personas mayores parecen ser mayores que los de las personas más jóvenes (Zaragoza et al., 2020). También se ha demostrado que el consumo de proteínas en las comidas durante todo el día puede influir en la masa muscular (Khoddam et al., 2019). De hecho, las intervenciones en las que las comidas que contienen menos proteínas se han "completado" con proteínas suplementarias han mostrado un aumento de la masa muscular en personas de mediana edad (Samuelsson et al. 2019).

\section{Situación COVID-19 Ecuador}

Según el Comité de Operaciones de Emergencia Nacional (2020), la cuarentena fue la medida ejecutada por el gobierno de la Republica del Ecuador desde el jueves 12 de marzo de 2020. La cuarentena fue la separación y restricción de movimiento de personas que potencialmente han estado expuestas al Covid-19, como enfermedad contagiosa, también para verificar y analizar si los individuos se sienten mal, reduciendo así el riesgo de que infecten a otras personas. Esta definición 
difiere del aislamiento, que es la separación de las personas a las que se les ha diagnosticado una enfermedad contagiosa de las personas que no están enfermas;

La ONU, (2020) en su plan de respuesta humanitaria Covid-19 Ecuador, aplica los términos aislamiento y cuarenta a menudo. La palabra cuarentena se usó por primera vez en Venecia, Italia en 1127 con respecto a la lepra y se usó ampliamente en respuesta a la peste negra, aunque no fue hasta 300 años después que el Reino Unido comenzó a imponer adecuadamente la cuarentena en respuesta a la peste. Más recientemente, la cuarentena se ha utilizado en el brote de la enfermedad por coronavirus 2019 (COVID-19).

Este brote ha hecho que ciudades enteras de Ecuador se coloquen efectivamente en cuarentena masiva, mientras que a muchos miles de ciudadanos extranjeros que egresaron de Ecuador se les ha pedido que se aíslen en sus hogares o en instalaciones estatales. Para 7 de septiembre de 2020 el ministerio de Salud Pública (2020), a través de la Plataforma Gubernamental de Desarrollo Social reporta que se han tomado 351.042 muestras para COVID-19 de las cuales 109.784 son casos confirmados con pruebas de Reacción en Cadena de Polimerasa (PCR).

Entre las principales dificultades que se presentan en el Ecuador por la pandemia Covid-19 se encuentran, la limitada capacidad de diagnóstico para la detección, aislamiento y atención oportuna de los casos. Falta de personal sanitario para las acciones de vigilancia epidemiológica y atención oportuna de casos en todos los niveles de atención. Escasez de equipos de protección personal (EPP), equipamiento e insumos a nivel nacional, así como de proveedores a nivel internacional para suplir la demanda. Dificultades para la expansión de los servicios de salud para la atención de pacientes que requieren hospitalización y cuidados críticos.

También se presentan dificultades para el manejo de cadáveres, debido al incremento del número promedio de fallecimientos por día y la reducción de la capacidad de las funerarias del sector público y privado. Prevención de infecciones hospitalarias para proteger al personal sanitario de eventuales contagios de COVID-19. En especial, los efectos socioeconómicos al enfrentar una paralización económica, social y política compleja ante la pandemia.

\section{Problemática de la investigación}

En orden de que las personas envejecen se producen cambios negativos. Negativos a nivel mental y fisiológico, todos estos conducen a muchos problemas para realizar las actividades diarias. Por ejemplo, adultos mayores tienen dificultades para caminar dos o tres cuadras o para trasladarse de la silla (Piasecki et al., 2016). También se presentan problemas con su marcha lenta cuando cruzan la calle antes de que cambie el semáforo. Otro grupo de consecuencias son las caídas y severas fracturas. Las estadísticas muestran que el 30\% de las personas mayores de 65 años, caen al menos una vez al año y en el grupo de mayores de 80 años y ancianos en hogares de ancianos, el número aumenta hasta un 50\% (Piasecki et al., 2016 y Donna et al., 2020). 
Partiendo de éste concepto se considera a las personas adultas mayores como vulnerables porque su organismo tarda más tiempo en recuperarse de cualquier proceso que afecte a su normal funcionamiento, es por ello que la situación de aislamiento social que viven actualmente a causa de la pandemia global por Covid-19 afecta con mayor fuerza a éste grupo etario (Donna et al., 2020). Ante ésta situación los adultos mayores conservan el distanciamiento social desde sus casas obligados a cambiar sus actividades cotidianas a una situación muy limitada de soledad, problemas emocionales, fisiológicos, sedentarismo, mala alimentación que provoca graves complicaciones en su salud y calidad de vida (Le Couteur y Anderson, 2020).

El statu QUO de los adultos mayores en estado de cuarentena ha generado que la actividad física sea muy limitada por la falta de espacios y situación de preocupación y angustia al permanecer en sus casas muchos de ellos solos o con familiares, cabe recalcar que el sedentarismo es la ausencia de actividad física; la misma perjudica a los individuos, ya que aumenta el número de enfermedades, como también contribuye a afectar el movimiento habitual reduciendo el disfrute de las experiencias comunes del hogar (Petretto y Pili 2020; Osama, Pankhania y Majeed 2020). El comportamiento sedentario se define como cualquier comportamiento de vigilia caracterizado por un bajo gasto de energía mientras se está sentado o recostado (Jensen et al., 2010).

Se ha informado que una mayor duración del comportamiento sedentario conduce a una mayor obesidad, diabetes tipo 2, disminución de la densidad ósea, enfermedades cardiovasculares y mortalidad (Jancey et al., 2009). Particularmente en el caso de las personas mayores, aproximadamente el $60 \%$ de sus horas de vigilia se pasan sentados, y son el grupo que tiene la mayor duración del comportamiento sedentario (Liping et al., 2020). Según la OMS (2015), siendo uno de los factores que influyen en el envejecimiento saludable, es el mantener buenos hábitos a lo largo de la vida, se centran en mantener una dieta equilibrada, realizar actividades físicas periódicas, ya que mantiene la masa muscular y por ende aporta a preservar la función cognitiva.

A nivel psicológico, el Ministerio de Salud Pública, (2020b) emitió los lineamientos operativos para la intervención de salud mental en la emergencia sanitaria del Ecuador, solicitando la participación y atención, de psicólogos clínicos, psicólogos infantiles y psicorehabilitadores; trabajadores sociales y psiquiatras en hospitales básicos, generales, especializados y de especialidades, el equipo y solicitud fue establecido según los lineamientos de salud mental hospitalaria, es decir: psiquiatra, psicólogo, trabajo social, terapista ocupacional, médicos y enfermeras capacitados en temas de salud mental.

La solicitud del Ministerio de Salud Pública, (2020b) fue emitida en torno a la prevención de trastornos leves o agudos de estrés emocional que puede generar el estado de cuarentena en la población. Para el Comercio (2020), en la población ecuatoriana se presenta frecuentemente una inestabilidad emocional, a través de estudios de teleasistencia digital, el ciudadano en cuarentena fue significativamente más propenso a informar agotamiento, desapego de los demás, ansiedad al tratar con pacientes febriles, irritabilidad, insomnio, falta de concentración e indecisión, deterioro del desempeño laboral y renuencia a trabajar o consideración por la resignación. La 
psico-afectividad también fue detectada en estudios en Ecuador por la falta de comunicación según Guerrero (2020); Gutiérrez, Cruz y Saldívar, (2020).

\section{Objetivo de la investigación}

Analizar la actividad física, alimentaria y psicológica emergente del estatus QUO en los adultos mayores del CEAM la Delicia en estado de cuarentena durante el lapso enero-mayo de 2020 por el Covid19-Ecuador. Para desarrollar la investigación en primer lugar se aplicó un análisis teórico documental sobre los estándares de alimentación y actividad física de la población mayor. Luego se aplicó una evaluación diagnostica para describir el statu quo alimenticio, físico y psicológico de la población mayor en el CEAM de la Delicia, Ecuador.

\section{METODOLOGÍA}

El estudio se desarrolló mediante la metodología multimétodo. Este método recomendado por Hernández, Fernández y Baptista (2014) permite aplicar los paradigmas cualitativos y cuantitativos. El sistema mixto o multimetodo brinda una mayor transparencia en la investigación, facilitando mejores alcances y resultados en las ciencias sociales y naturales (Büthe y Jacobs, 2015).

\section{Diseño de la investigación}

El estudio se estructuro con el diseño exploratorio secuencial (Dexplos), el mismo implica una primera con la recolección de información y análisis de índole cualitativo, considerado la primera fase. La fase cualitativa es de tipo teórico - documental, que sirve como complemento y extensión de la investigación biográfica. Los autores investigan, analizan y adquieren diferentes significados en el campo de la salud.

En cierto sentido, la investigación documental se convirtió en sinónimo de investigación de archivo digitalizado, es decir no se pudo cuestionar relacionadas con el papel, libros impresos, uso de documentos impresos, registros públicos o privados, ya que los autores del estudio no pueden movilizarse o salir de la residencia o las bibliotecas más cercanas por motivos de movilidad y resguardo de la cuarentena. La segunda fase, fue cuantitativa con la recolección de datos y análisis descriptivo de los datos (Büthe y Jacobs, 2015).

\section{Población y muestra}

La población del estudio estuvo establecida por la comuna de La Delicia, con un total de 6700 habitantes en la ciudad de Quito, Republica del Ecuador. La muestra fue de selección aleatoria no probabilística de tipo intencional. Este método de muestreo admite seleccionar casos particulares de una población restringiendo la muestra sólo a determinados casos. Se recurre a espacios o ambientes de estudios donde la población es de gran variabilidad y por lo tanto la muestra no supera los 10000 habitantes o sujetos generalizados (Otzen y Manterola, 2017). 
De igual forma, para la selección de la muestra se aplicaron los estándares de la Ley Orgánica de las Personas Adultas Mayores (2019) que constituyen un adulto mayor, quien posea 65 años de edad o más. En anexo a la selección, también fueron establecidos los adultos que se encuentran registrados en el Centro de la experiencia del adulto mayor (CEAM) de la comunidad de la Delicia, con un total de la muestra de 115 personas.

\section{Instrumentos de la investigación}

Los documentos de evaluación generalmente incluyeron cuatro criterios: autenticidad, credibilidad, representatividad y significado (Scott, 2006). La autenticidad de los materiales, recae en la búsqueda de autores con estudios publicados en libros y revistas indexadas de alto impacto.

Se aborda si los materiales son genuinos o de origen cuestionable, y si su producción es original y confiable y no ha sido alterada posteriormente. Los investigadores, indagaron en identificar claramente que no existan alteraciones en las fuentes teóricas. La autenticidad generalmente se ve como el criterio más fundamental para toda investigación documental en la salud, ya que la confirmación de la autoría, el lugar y la fecha generalmente se determinan antes de que cualquier investigador continúe trabajando con el documento.

Para la recolección de datos en la fase cualitativa se aplicó la revisión teórico - documental de diferentes autores y estudios actuales sobre la población adulta, causas u efectos de la cuarentena en la actualidad del Covid-19. De forma digital exclusiva se dio uso a la revisión de diversas sesiones de videoconferencias por plataforma Zoom que permitieron visualizar las actividades de los coordinadores del CEAM con los adultos mayores durante las actividades. Estas fuentes digitales fueron evaluadas y analizadas por las investigadoras para interpretar los comportamientos actitudinales de los participantes.

Posteriormente en la fase cuantitativa se aplicó como instrumento de recolección de datos un cuestionario de opción múltiple con 15 ítems (Ver tabla 1). Mediante el proceso de indagación deductiva los investigadores interactuaron a distancia con los participantes y con los datos, buscando respuestas a preguntas que se centran en la experiencia social, cómo se crea y cómo da significado a la vida humana.

Los autores diseñaron el cuestionario en base a dos variables de estudio, la alimentación y las actividades físicas, pero estas variables a nivel cualitativo también permitieron analizar los aspectos psicológicos y actitudinales de los participantes. La encuesta como técnica de estudio, fue dirigida a los adultos mayores del CEAM a través del correo personal que poseen los usuarios registrados. Los encuestados solo debían elegir la opción considerada de forma personal. 
Tabla I. Cuestionario aplicado a los adultos mayores del CEAM La Delicia.

Encuesta del nivel de funcionalidad cotidiana en el adulto mayor CEAM La Delicia

Universidad Iberoamericana del Ecuador UNIB.E

Objetivo: Analizar el nivel de funcionalidad cotidiana para mejorar la calidad de vida en el Adulto Mayor del Centro de Atención Integral La Delicia D.M Quito.

Instrucciones: Lea la pregunta cuidadosamente / Responder todas las preguntas.

Datos _ Edad:___ Género: $\mathrm{M}$ :___ / F:___ Estado civil:

Cuestionario aplicado a los participantes del CEAM - La Delicia - Quito

\begin{tabular}{|c|c|c|c|c|c|c|}
\hline \multirow{2}{*}{$\begin{array}{l}N^{\circ} \\
1\end{array}$} & Ítems & \multicolumn{5}{|c|}{ Opciones } \\
\hline & $\begin{array}{l}\text { ¿Durante qué tiempo } \\
\text { realiza la actividad } \\
\text { recreativa? }\end{array}$ & $\begin{array}{l}\geq 40 \\
\text { minutos }\end{array}$ & $\begin{array}{l}\quad \leq 30 \\
\text { minutos }\end{array}$ & $\begin{array}{l}\leq 20 \\
\text { minutos }\end{array}$ & $\leq 10$ minutos & 0 minutos \\
\hline 2 & $\begin{array}{l}\text { ¿Cuantos días de la } \\
\text { semana realiza actividad } \\
\text { recreativa? }\end{array}$ & $\geq 4$ días & 3 días & 2 días & 1 día & 0 día \\
\hline 3 & $\begin{array}{l}\text { ¿Cuál es su nivel de } \\
\text { funcionalidad cotidiana? }\end{array}$ & Hiperactivo & Muy activo & Activo & $\begin{array}{l}\text { Poco } \\
\text { sedentario }\end{array}$ & Sedentario \\
\hline 4 & $\begin{array}{l}\text { ¿Cómo se } r \text { siente } \\
\text { anímicamente } \\
\text { de haego } \\
\text { recreativa? }\end{array}$ & Feliz & Alegre & Normal & Desmotivado & Deprimido \\
\hline 5 & $\begin{array}{l}\text { ¿En qué lugar realiza } \\
\text { actividad recreativa? }\end{array}$ & Casa & parque & Aire libre & $\begin{array}{l}\text { Centro de } \\
\text { recreación }\end{array}$ & No aplica \\
\hline \multirow[t]{2}{*}{6} & $\begin{array}{l}\text { ¿Qué tipo de actividad } \\
\text { recreativa realiza }\end{array}$ & $\begin{array}{l}\text { Bailo- } \\
\text { terapia }\end{array}$ & $\begin{array}{l}\text { Caminata a } \\
\text { distancia }\end{array}$ & $\begin{array}{l}\text { Caminar a } \\
\text { paso ligero }\end{array}$ & $\begin{array}{l}\text { Caminar a } \\
\text { paso ligero }\end{array}$ & ninguno \\
\hline & Gimnasia aeróbica & & & & & \\
\hline 7 & $\begin{array}{l}\text { ¿Cuántas veces come en } \\
\text { el día? }\end{array}$ & $\geq 5$ & 4 & 3 & 2 & 1 \\
\hline 8 & $\begin{array}{l}\text { ¿Cuántos litros de agua } \\
\text { toma en el día? }\end{array}$ & 3 litros & 2 litros & 1.5 litros & 1 litro & $1 / 2$ litro \\
\hline 9 & $\begin{array}{l}\text { Para usted la comida más } \\
\text { importante en el día es: }\end{array}$ & Desayuno & Aperitivos & Almuerzo & Merienda & Cena \\
\hline 10 & $\begin{array}{l}\text { ¿Los alimentos que usted } \\
\text { consume en el día son? }\end{array}$ & $\begin{array}{c}\text { Muy } \\
\text { saludables }\end{array}$ & Saludable & $\begin{array}{c}\text { Algo } \\
\text { saludable }\end{array}$ & $\begin{array}{l}\text { Poco } \\
\text { saludables }\end{array}$ & $\begin{array}{l}\text { Nada } \\
\text { saludables }\end{array}$ \\
\hline \multirow[t]{2}{*}{11} & ¿Qué grupo de & Grupo 1 & Grupo 2 & Grupo 4 y 5 & Grupo 6 & Grupo 7 \\
\hline & $\begin{array}{l}\text { alımentos consume } \\
\text { con más frecuencia en } \\
\text { cuarentena? }\end{array}$ & $\begin{array}{l}\text { Leche y } \\
\text { derivados }\end{array}$ & $\begin{array}{l}\text { Carnes, } \\
\text { pescados y } \\
\text { huevos }\end{array}$ & $\begin{array}{l}\text { Frutas, } \\
\text { vegetales, } \\
\text { verduras } \\
\text { Hortalizas }\end{array}$ & $\begin{array}{l}\text { Cereales y } \\
\text { derivados, } \\
\text { azúcar y } \\
\text { dulces }\end{array}$ & $\begin{array}{l}\text { Grasas, aceite } \\
\text { y mantequilla } \\
\text { Carbohidratos }\end{array}$ \\
\hline 12 & $\begin{array}{l}\text { ¿Considera que su } \\
\text { alimentación diaria es? }\end{array}$ & Adecuada & $\begin{array}{c}\text { Poco } \\
\text { adecuada }\end{array}$ & normal & Baja & Muy Baja \\
\hline
\end{tabular}

\section{Técnicas de análisis de los resultados}

Los resultados obtenidos en la fase cualitativas fueron analizados de forma interpretativa. Se inició el estudio con un análisis de tipo documental como técnica de investigación cualitativa. Así Shakouri1 (2014), argumenta que debe existir la honestidad, la coherencia y correspondencia de los temas investigados con los sustentos teóricos, ya que son una base valida y sostenible de 
los estudios interpretativos. Los sustentos teóricos analizados en la primera fase, conllevan a la segunda fase cuantitativa de la investigación. De esta forma los datos obtenidos se analizan de manera descriptiva, mediante la revisión estadística de ponderación o porcentajes.

Los resultados de ambas fases fueron trianguladas o contrastadas. Given (2008), recomienda la triangulación de datos, de esta forma se implica obtener datos de diferentes fuentes, o en diferentes momentos o bajo diferentes condiciones. Las condiciones, no incluyen los estudios en los que éstos comprenden las variables independientes. En la siguiente sección se contrastan los resultados cualitativos y cuantitativos del estudio.

\section{RESULTADOS Y DISCUSIÓN}

Los resultados obtenidos de índole cualitativo y cuantitativo, permiten analizar y caracterizar el estado de vida del adulto mayor del CEAM de La Delicia en Ecuador, durante la situación de cuarentena en el lapso enero - mayo 2020. De esta forma analizar relación entre el ejercicio físico, la alimentación y la calidad de vida. Para ello se tomó en cuenta la variable actividad física con los factores, tiempo, estado emocional, y ambiente de desempeño de la actividad física. Seguidamente la variable alimentación, compuesta por el factor, frecuencia alimentaria, importancia alimentaria y clasificación de alimentos.

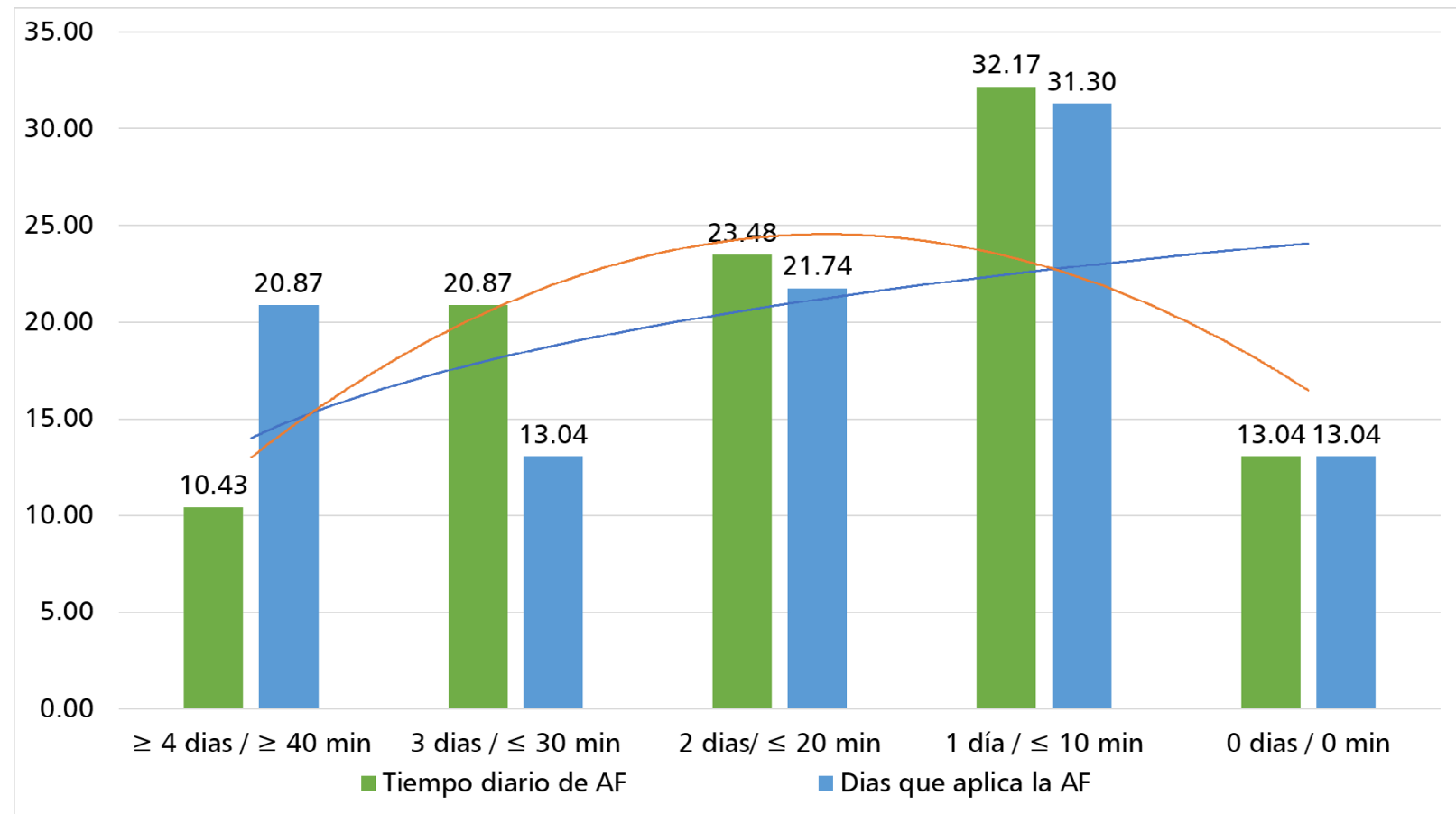

Gráfico. 1. Resultados de la variable actividad física (AF), factor "Tiempo" del cuestionario aplicado a los adultos mayores del CEAM

En base a los resultados se puede demostrar que su mayoría el 32,17\% y 31,30\% de los encuestados manifiestan realizar AF en promedio de un día a la semana con diez minutos. Luego un 23,48\% de AF en dos días a la semana con 20 minutos, también de un 20,87\% con el desempeño tres días de AF con un 13,04\% de 30 minutos de ejercicios. Entre los valores porcentuales más críticos se encuentra un $13,04 \%$ de los adultos mayores que no aplican AF, (Gráfico. 1). 
La dosis de AF o ejercicio se describe por la duración, frecuencia, intensidad y modo (Latham y Liu, 2010). Para obtener efectos óptimos, la persona o adulto mayor debe cumplir con el programa de ejercicio prescrito y seguir el principio de sobrecarga del entrenamiento, es decir, hacer ejercicio cerca del límite de la capacidad máxima para desafiar los sistemas del cuerpo lo suficiente, para inducir mejoras en los parámetros fisiológicos como VO2max y fuerza muscular (Taylor, 2014).

Las mejoras en la salud mental, el bienestar emocional, psicológico y social y la función cognitiva también se asocian con la AP regular. A pesar de estos beneficios para la salud, los niveles de AP entre los adultos mayores permanecen por debajo de los $150 \mathrm{~min} / \mathrm{semana}$ recomendados por (Boulton, Horne y Todd, 2018). La cruda prevalencia global de inactividad física es del 13,04\% (ver Gráfico 1). Esto se traduce en que uno de cada diez adultos está físicamente inactivo, también 5 de cada diez con niveles de actividad inferiores a las recomendaciones actuales de (OMS, 2010).

La inactividad y el envejecimiento aumentan el riesgo de enfermedades crónicas, y las personas mayores a menudo tienen múltiples afecciones crónicas. Las recomendaciones de ejercicio de OMS (2010) incluyen ejercicios aeróbicos y ejercicios de fuerza, así como ejercicios de equilibrio para reducir el riesgo de caídas. Si los adultos mayores no pueden seguir las pautas debido a afecciones crónicas, deben ser tan activos como lo permitan su capacidad y condiciones (Osama, Pankhania y Majeed, (2020). Es importante tener en cuenta que la cantidad recomendada de AF se suma a las actividades de rutina de la vida diaria, como el cuidado personal, la cocina y las compras, por mencionar algunas.

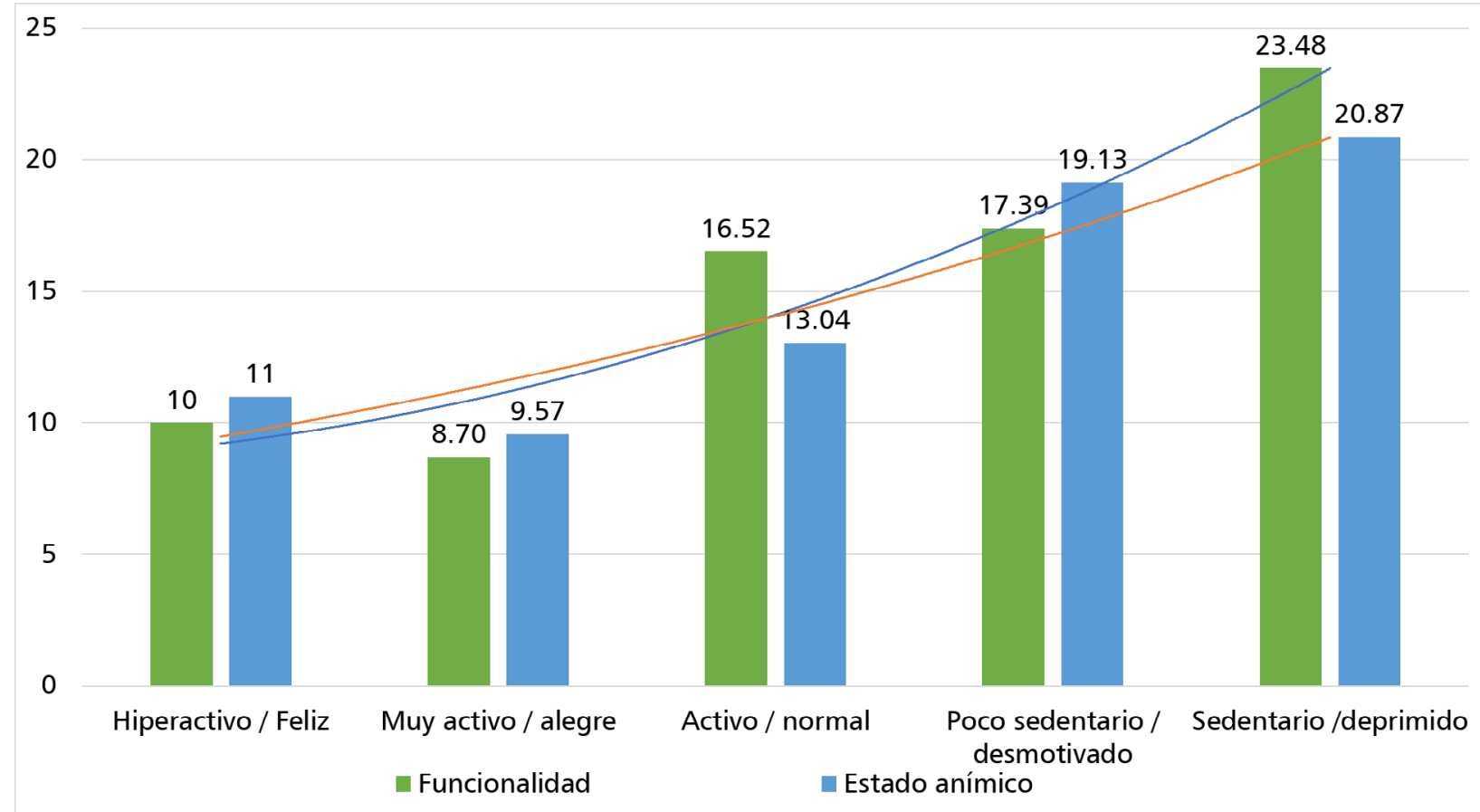

\section{Gráfico. 2 Resultados de la variable actividad física (AF), factor "Estado emocional" del cuestionario aplicado a los adultos mayores del CEAM}

La selectividad socioemocional es una teoría de la motivación de por vida que predice una mayor regulación de las emociones con la edad (Ready, Åkerstedt y Mroczek, 2011). Según la teoría, la perspectiva del tiempo es la fuerza dominante que estructura las motivaciones y objetivos 
humanos. La teoría sostiene que los humanos tienen una conciencia consciente y subconsciente de su tiempo restante en la vida, y que los límites percibidos en el tiempo dirigen la atención a los aspectos emocionalmente significativos de la vida. Cuando el tiempo se percibe como expansivo, como sucede en los adultos jóvenes sanos, el esfuerzo por alcanzar los objetivos y las motivaciones relacionadas se centran en la obtención de información.

Se valora la novedad y se realizan inversiones para ampliar los horizontes. En contraste, cuando el tiempo se percibe como limitado, la experiencia emocional asume la primacía; las personas están motivadas para monitorear y seleccionar sus entornos para optimizar el significado emocional y el funcionamiento emocional. Según la teoría, y respaldada por la evidencia cuantitativa se observa que el $23.48 \%$ de las personas que viven en un lugar establecido y $20,87 \%$ de los encuestados manifestaron estar deprimidos (Gráfico. 2).

Los adultos mayores manifiestan estos cambios motivacionales similares debido al tiempo de inactividad e inmovilidad de la cuarentena, considerando que su límite de tiempo se acorta a nivel mental o psicológico, para la mayoría en parte, al tiempo que queda en la vida, la teoría de la selectividad socioemocional plantea que la regulación de los estados emocionales recibe mayor prioridad a medida que las personas adultas o mayores conviven o se relacionan con ciudadanos y ejercen el traslado o movimiento fisiológico, como por ejemplo el realizar a visita de amistades o familiares (Grühn, Sharifian y Chu, 2016).

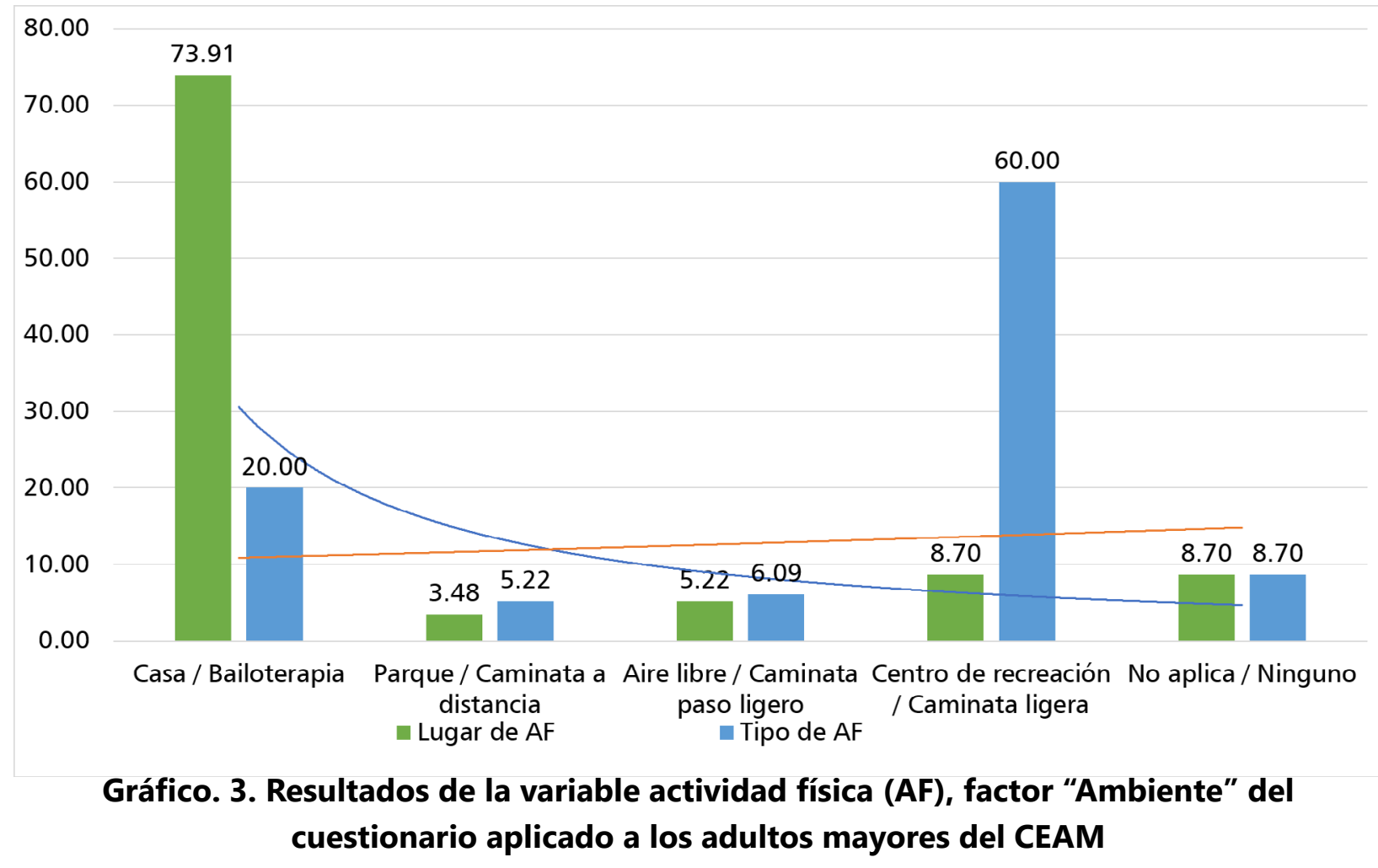

Para Bendayan et al., (2020) el envejecimiento saludable se define como la capacidad de llevar un estilo de vida saludable, socialmente inclusivo, relativamente libre de enfermedades o discapacidades y esto es más probable en aquellos que participan activamente en actividades para mejorar su salud y bienestar del CEAM. Los resultados demuestran que $73,91 \%$ y un $60 \%$ de 98 
los encuestados se mantienen en su hogar y aplican AF de caminatas ligeras, seguidamente de un $20 \%$ que aplican bailoterapia como AF (Gráfico. 3). Los datos señalan que existen dificultades obvias con las actividades habituales de la vida diaria, la gran mayoría de las personas mayores de 65 años tenían una función fisiológica más baja que los adultos jóvenes.

En visión post hipotética, el permanecer tanto tiempo en el hogar, conlleva a que los adultos mayores tengan un índice de masa corporal más alto debido al aumento de la gordura, con músculos más pequeños y más débiles, particularmente en las piernas, menor densidad mineral ósea, función cardiorrespiratoria y metabólica reducida y peor desempeño en pruebas cognitivas según (Bijlsma et al., 2013). Otros estudios han demostrado que al estar de forma sedentaria en el hogar sin AF, se genera entre un 30\% y 50\% menos neuronas motoras que inervan los músculos de las piernas de ancianos sanos en comparación con jóvenes, lo que sugiere que la remodelación de la unidad motora es parte del proceso normal de envejecimiento (Campbell, McComas y Petito, 1973).

Las pérdidas de la neurona motora y la fibra muscular que ocurren durante el envejecimiento nunca pueden ser reemplazadas, pero la estructura y función de los sistemas cardiorrespiratorio, metabólico y musculo esquelético son susceptibles de mejorar a través de la AF, por lo que es informativo para un perfil muy atlético de personas mayores (Lexell, Taylor y Sjöström, 1988).

Las disminuciones progresivas en la función fisiológica que generalmente ocurren durante décadas se asocian con una velocidad de caminata más lenta y dificultades para levantarse de una posición sentada y equilibrarse. Se han desarrollado evaluaciones estandarizadas para indicar la capacidad física en la edad avanzada, incluida la caminata de 6 minutos y la prueba de elevación de la silla de 30 segundos según (Jones, Rikli y Beam, 1999).

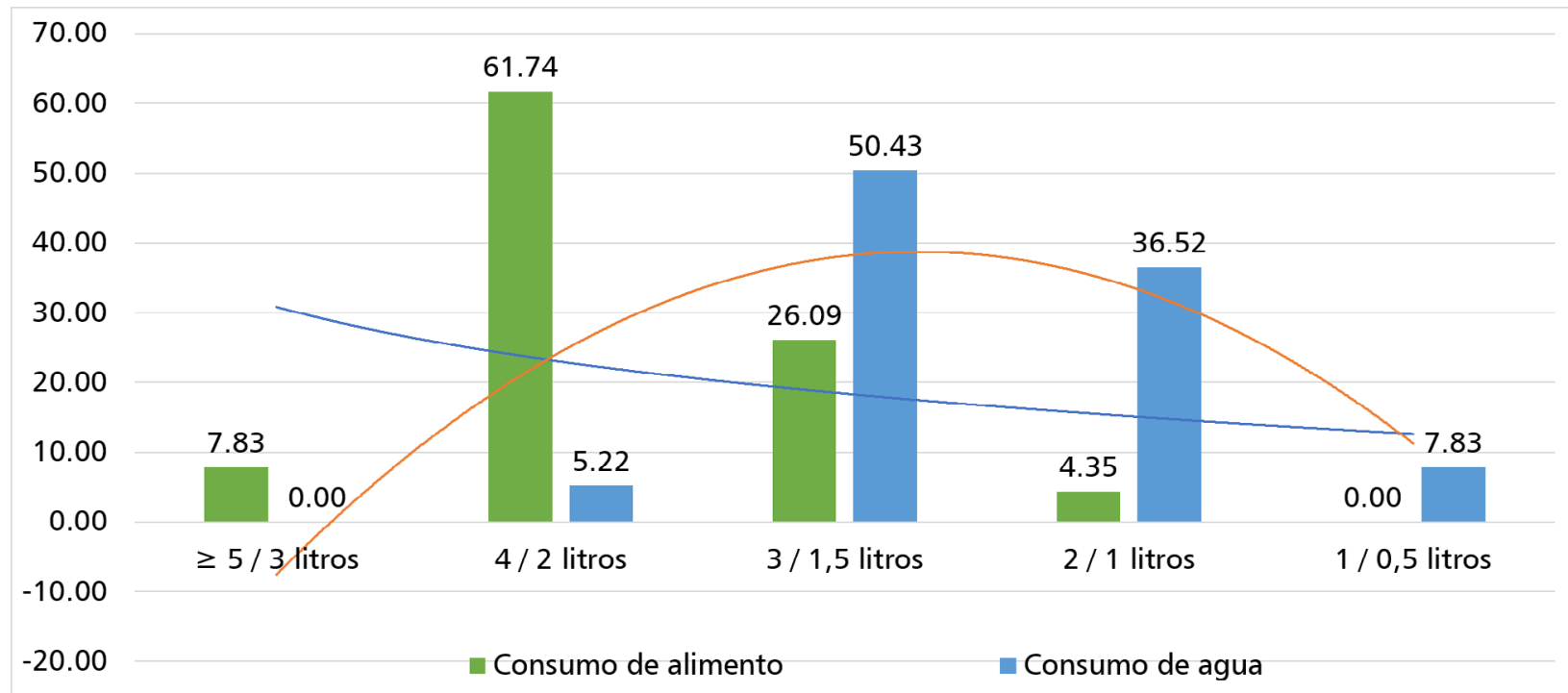

Gráfico. 4. Resultados de la variable alimentación (A), factor "frecuencia alimenticia" del cuestionario aplicado a los adultos mayores del CEAM 
El grafico 4, representa la variable alimentación factor frecuencia que establecen los adultos mayores del CEAM La Delicia, los resultados de frecuencia de consumo diario demuestran que en su mayoría el 61,74\% de los encuestados consumen alimentos 4 veces al día, aunado al porcentaje más alto de consumo de agua 1,5 litros con un 50,43\% de los encuestados. Seguidamente el 26.09\% de los adultos mayores indicaron consumir 3 veces al día su alimentación y el segundo porcentaje de consumo de agua en 36,52\% con un volumen aproximado de 1 litro.

En la actualidad, la cuarentena está relacionada a un stop de vida, esto podría trascender en un status de fastidio no solo para los encuestados sino para todos sus familiares o con quienes conviven. El aburrimiento e inmovilidad se asocia con ingerir mayor cantidad de alimentos, mayor nivel de consumo de grasa, carbohidrato y proteína según (Moynihan et al., 2015). Además, durante la cuarentena, escuchar o leer noticias de forma frecuente y continua sobre la pandemia sin interrupción puede ser estresante. En consecuencia, Yılmaz y Gökmen (2020) afirman que el estrés conduce y motiva a las personas a comer en exceso, principalmente buscando en búsqueda de azúcar o al consumo de alimentos reconfortantes.

Como se puede percibir en la imagen 2, los adultos mayores, resaltaron encontrarse desmotivados y deprimidos. Estos niveles emocionales, generan el apetito de consumir un cierto tipo de provisiones, definiéndose como monomanía de alimentos, es decir, una concepción múltiple que contiene terminologías emocionales como el anhelo acelerado de digerir, a nivel conductual se refleja en la búsqueda de alimento, de igual forma, el ser se encuentra en un proceso cognitivo sobre la dispensa de comida en su hogar, en cuanto el estatus fisiológico se percibe en la expectoración o salivación (Rodríguez y Meule, 2015).

En cuanto al consumo de agua, los investigadores analizaron documentos que exponen que el agua potable puede prevenir el coronavirus al mantener la boca y la garganta húmedas según (Koman, 2020 y Barry, Kristen, y Irwin, 2010). Según Jin, Cai y Cheng (2020) esto no es cierto, aunque el simple acto de beber agua no evitará contraer COVID-19, hay otras formas en que beber más agua pueda mejorar en el estado de hidratación durante el presente brote a los adultos mayores. Para empezar, el cuerpo necesita agua para funcionar. El aumento de la ingesta de agua puede hacer que su cuerpo procese de manera más eficiente, lo cual es importante para el sistema inmunológico (Koman, 2020). Beber agua también puede mejorar la función cognitiva, lo que hace más consciente en el comportamiento, lo que podría conducir a decisiones más saludables y seguras.

De igual forma, consumir agua hará un mejor sistema digestivo, facilitando la frecuencia de asistir al baño. Ahora, es importante señalar que hay formas en que beber demasiada agua en realidad puede ser perjudicial para su salud. La sobre-hidratación puede provocar que los niveles de sodio en el cuerpo disminuyan debido a la sobresaturación. Si bien no existe una ciencia exacta para determinar cuánta agua necesita consumir durante el día, lo importante es asegurarse de que esté bebiendo agua, pero no de forma exacerbada por más de 1,5 litros diarios, ya que el cuerpo no va a digerir de forma adecuada el exceso de líquido (OMS, 2020). 


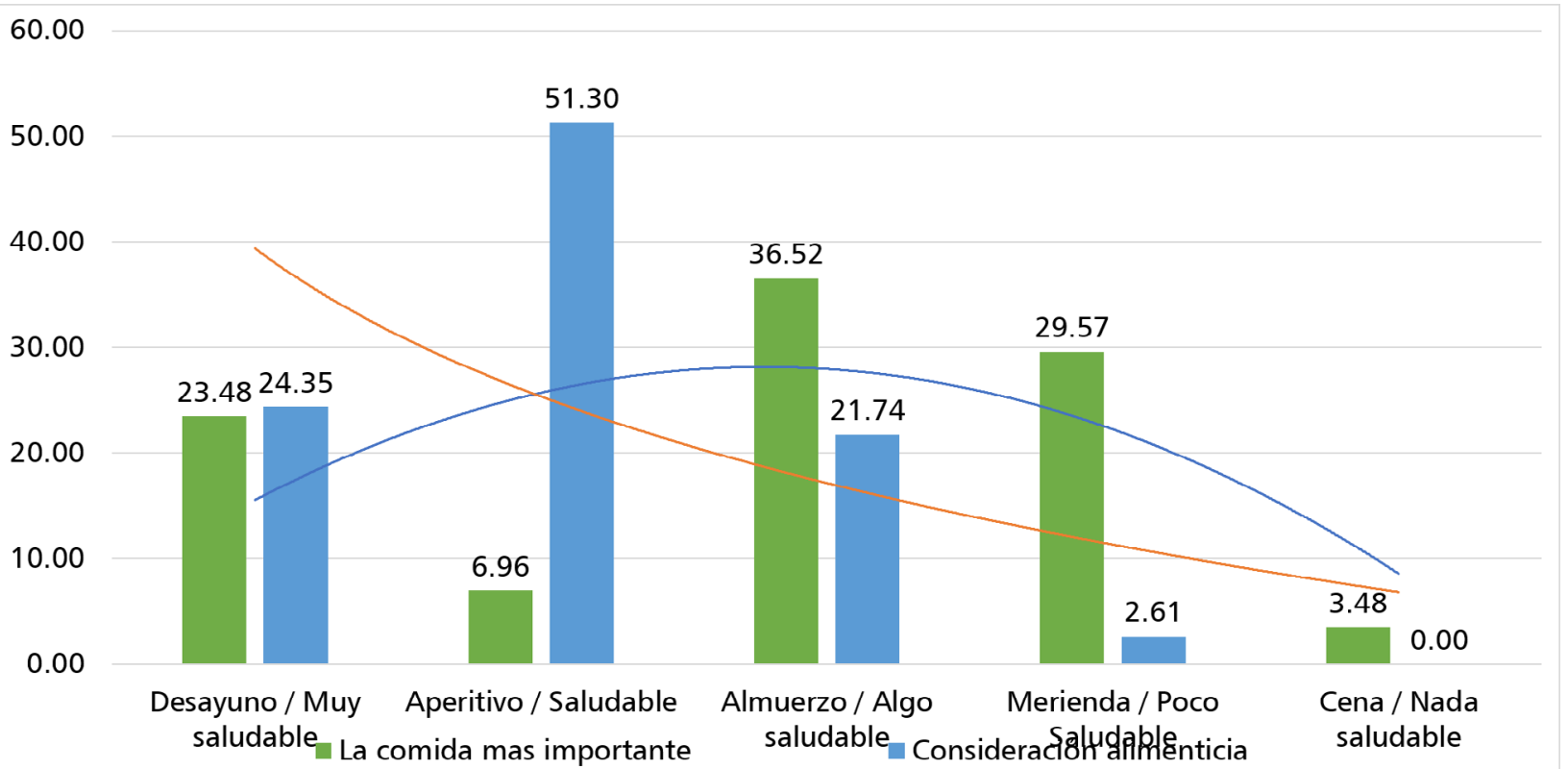

\section{Gráfico. 5. Resultados de la variable alimentación (A), factor "importancia alimenticia" del cuestionario aplicado a los adultos mayores del CEAM}

Entre los resultados expuestos en el gráfico 5, demuestra que los encuestados consideran que el alimento más importante es el almuerzo con un 36,52\% de acordados, considerado como alimento saludable, luego el alimento más importante es la merienda con un 29,57\% de aceptación y se considera saludable por un 24,35\% de los adultos mayores de CEAM (Imagen 5). Para Liping et al., (2020) se considera el desayuno como el alimento más importante, en estudios se considera que, en adultos de 65 a 82 años durante un período de 16 años, se comprobó quienes saltaron el desayuno tenían un $27 \%$ más de riesgo de ataque cardíaco o muerte por enfermedad cardíaca que aquellos que honraron la comida de la mañana.

Según los científicos Liping et al., (2020), saltarse el desayuno puede hacer que tenga más hambre y más probabilidades de comer comidas más grandes, lo que conduce a un aumento en el azúcar en la sangre. Tales picos pueden allanar el camino para la diabetes, la presión arterial alta y los niveles altos de colesterol, todos factores de riesgo que pueden convertirse en un ataque cardíaco.

WaldroupH y Hellwig (2000) recomiendan no exceder en el consumo de alimentos en horas de la tarde. Las calorías se queman sin importar cuándo las coma, por lo que teóricamente está bien comer después del anochecer. Pero si come una cena abundante, no es probable que se eliminen esas calorías antes de dormir. Las calorías que no se queman son más probables en almacenarse como grasa, ya que se el cuerpo se vuelve menos activo hacia el final del día. De igual para los autores citados, digerir alimentos cerca de la hora de acostarse, aumenta el azúcar en la sangre y la insulina, lo que hace que le resulta difícil quedarse dormido. Por lo tanto, la última comida debe ser la más ligera del día y debe comerse al menos tres horas antes de irse a dormir. 


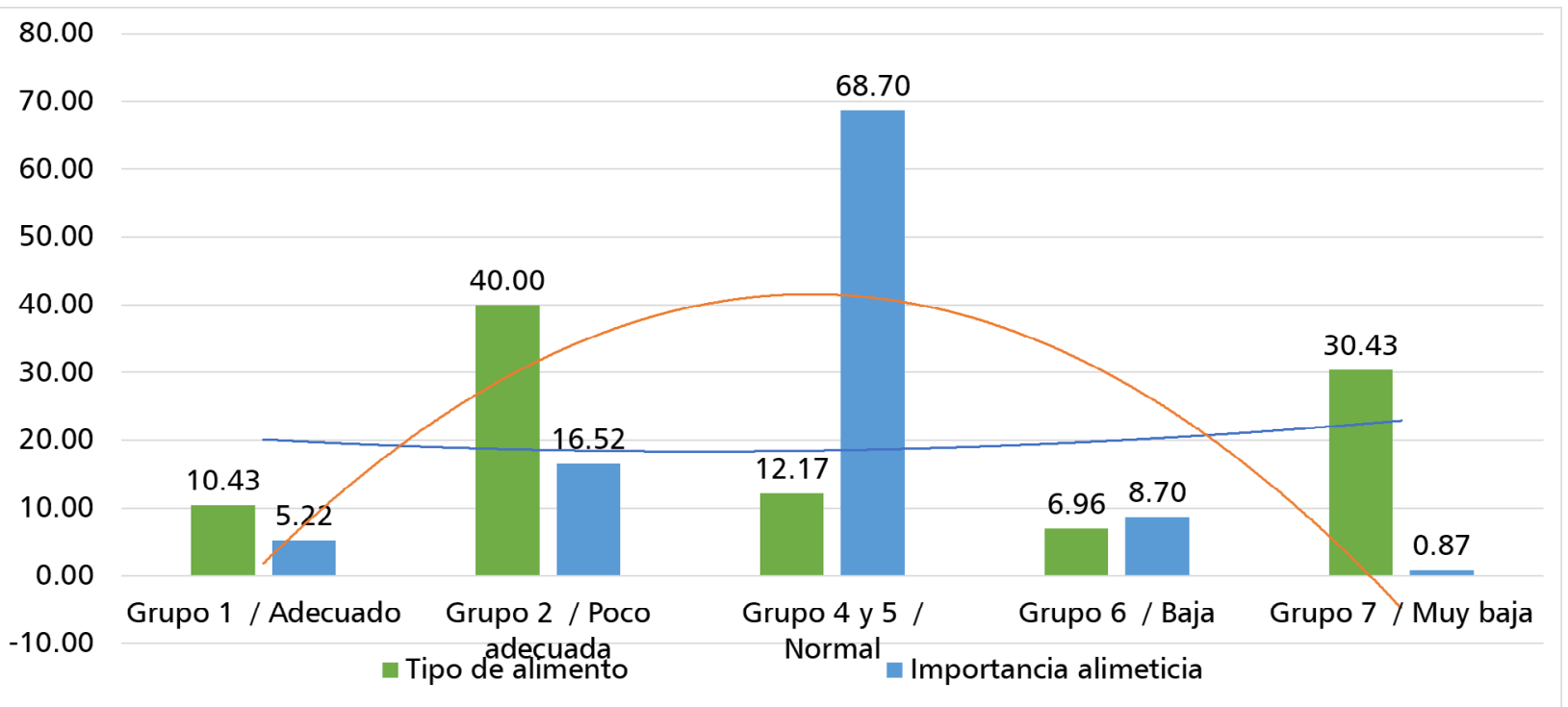

Gráfico. 6. Resultados de la variable alimentación (A), “clasificación de alimento" del cuestionario aplicado a los adultos mayores del CEAM

En el Gráfico. 6, los encuestados manifestaron consumir con mayor frecuencia alimentos del grupo 2 con un $40 \%$, según Calañas (2005), corresponde a carnes, pescado y huevos. Seguidamente de alimentos del grupo 7 con un $30,43 \%$ de consumo. En la comida típica tradicional ecuatoriana, se encuentran alimentos como el cerdo o chancho, pavo, gallinas, res y pescado, considerados en la alimentación de los adultos mayores como normal por un $68,70 \%$ de soporte. De esta forma según, los datos para Power et al., (2014) los adultos mayores que viven de forma independiente tienen déficits en la ingesta de vitamina $C$ y calcio más, vitamina $D$, ácido fólico, zinc y magnesio.

Jensen et al., (2010) exponen que el déficit en la ingesta de micronutrientes se encuentra acompañado por una alta prevalencia de sobrepeso y obesidad (70\%), lo que sugieren en aplicar ingestas de alimentos densos en energía, pero pobres en micronutrientes en este grupo. El problema de la ingesta baja de micronutrientes se destaca con el ejemplo de la vitamina D.

Por lo tanto, durante este tiempo es importante cuidar los hábitos nutricionales, siguiendo un patrón nutricional saludable y equilibrado que contenga una gran cantidad de minerales, antioxidantes y vitaminas. Thurnham (1997) hace énfasis en que la ingesta de frutas y verduras (grupo 4 y 5) suministran suficientes micronutrientes que acrecientan la función inmunológica en los adultos mayores. Lo mencionado acontece porque ciertos micronutrientes como la vitamina $\mathrm{E}_{\text {, }}$ la vitamina $\mathrm{C}$ y el betacaroteno poseen antioxidantes.

En el aspecto socio emocional durante las sesiones aplicadas por el CEAM los investigadores percibieron que los participantes reportaron aspectos actitudinales negativos como el miedo, nerviosismo, tristeza y sentido de culpabilidad. Pocos participantes demostraron sentimientos positivos, sentimiento como la felicidad o la tranquilidad. Las autoras del estudio lograron percibir durante las sesiones comportamientos como la confusión, el enfado, el dolor, entumecimiento e insomnio inducido por ansiedad. 
Otro aspecto de habito adquirido por los participantes fue el comportamiento a largo plazo, después del primer mes de inicio de cuarentena, muchos participantes notificaron que sus hijos y familiares no consideraron como relevante o de precaución el contagio del Covid-19. Después de anunciarse un alto índice de casos, reportes de contagiados y muertos en la ciudad de Quito, los adultos mayores consideraron tomar medidas preventivas como el uso de mascarilla, lavarse las manos con atención y evitar las multitudes, para algunos, adaptarse a las actividades del CEAM con precauciones de contagiarse en la pandemia es difícil.

\section{CONCLUSIONES}

Después de aplicar la descripción de actividad física, alimentaria y análisis psicológico de los encuestados del CEAM La Delicia, se puede concluir que el sedentarismo provoca que los adultos mayores la perdida y disminución de la funcionalidad efectiva de una manera más acelerada. La inmovilidad o sedentarismo genera enfermedades en el sistema cardiovascular, sistema óseo y la masa muscular. La cuarentena produce un estrés que tiene afinidad al insomnio o trastornos de sueño, el cual genera un vínculo de ansias alimentarias. Las AF, otorga a los adultos mayores la eventualidad de mejorar el ciclo de vida.

Con el desarrollo de ejercicios o AF se perciben cambios tangibles a nivel fisiológico entre estos, el sistema muscular, con una mejor tonificación, mejoría en el equilibrio, mayor flexibilidad, se optimiza la resistencia cardiaca. Conjuntamente, estas actividades facilitan redimir la depresión, el miedo, el temor, el pánico, entre otros. Para que los cuerpos de un adulto mayor tengan un patrón de azúcar balanceado en la sangre, se recomienda una alimentación en fibras, tostadas de avena y trigo integral con mantequilla de almendras, o una tortilla con espinacas y aguacate (Elia, 2000). Alimentarse con tostadas, panes que incluyan granos germinados o molidos en piedra y que enumeren los granos enteros como uno de los primeros elementos en una etiqueta de ingredientes. Los granos pueden hacer que el pan sea más denso y abundante.

De igual forma, se recomienda el consumo bajo de carbohidratos, aunque estos fomentan la obtención de serotonina esta tiene un resultado positivo al estabilizar los estados de ánimo en los adultos mayores. En cierto sentido, los alimentos ricos en carbohidratos pueden ser una forma de auto-medicarse contra el estrés. El efecto del deseo de carbohidratos en el bajo estado de ánimo de los adultos mayores es proporcional al nivel de glucemia de la comida. Pero esta rutina nutricional perniciosa puede elevar el riesgo de sustentar la obesidad, como también establecer enfermedades cardiovasculares, dificultad respiratoria y síntomas diabéticos que están relacionados con los indicios más frecuentes que se presentan en los pacientes infectados con CoVID-19 (Wu et al., 2020).

Entre los aspectos psicológicos mas evidentes por los participantes fue el miedo a la infección, demostrando el temor sobre su propia salud o temores de infectar a otros. Durante los brotes importantes de enfermedades infecciosas, la cuarentena puede ser una medida preventiva necesaria. Sin embargo, las autoras sugieren que la cuarentena se asocia con un efecto psicológico 
negativo en los adultos mayores como la impotencia social para afrontar la situación según Jeong, Yim y Song (2016).

Durante el período de cuarentena, este efecto psicológico negativo no es sorprendente, sin embargo, la evidencia de que un efecto psicológico de la cuarentena aún puede detectarse meses o años después, Liu, Kakade y Fuller, (2012) expresan que los efectos post pandemia son más preocupantes y sugieren la necesidad de asegurar que se implementen medidas de mitigación efectivas como parte del proceso de planificación de cuarentena.

Finalmente se puede analizar que el entrenamiento físico, la alimentación sana en fibras y vitaminas en personas mayores se asocia a los beneficios para la salud. La AF y la alimentación son factores claves en la prevención primaria, secundaria y terciaria del manejo de enfermedades, así como para mejorar el rendimiento físico y las actividades de la vida diaria. Los investigadores, recomiendan promover el ejercicio en la población de edad avanzada ya que el mismo se considera un problema clínico y de salud pública. Sin embargo, privar a las personas de su libertad por el bien público en general suele ser polémico y debe manejarse con cuidado. Si la cuarentena es esencial, los resultados sugieren que los funcionarios deben tomar todas las medidas para garantizar que esta experiencia sea lo más tolerable posible para las personas.

\section{REFERENCIAS BIBLIOGRÁFICAS}

Andrade, J., Do Nascimento, J., Sousa, L., Morais, G., Oliveira D., Fiorese, L. \& Lorcan, D. (2020). Cross-Cultural Adaptation and Psychometric Properties of the Portuguese Version of the Life Skills Scale for Sport. Measurement in Physical Education and Exercise Science, 24(1), 11-24. DOI: 10.1080/1091367X.2019.1647208

Barry, M., Kristen, E. y Irwin, H. (2010). Agua, hidratación y salud. Nutrition Reviews, 68(8), 439-458. Doi: https://doi.org/10.1111/j .1753-4887.2010.00304.x

Bendayan, R., Kelly, A., Hofer, S. M., Piccinin, A. M., y Muniz-Terrera, G. (2020). Memory Decline and Depression Onset in U.S. and European Older Adults. Journal of Aging and Health, 32(3), 189-198. DOI: https://doi.org/10.1177/0898264318813019

Bijlsma, A.Y., Meskers, M.C., Molendijk, M., Westendorp, R.G., Sipilä, S., Stenroth, L., Sillanpää, E., McPhee, J.S., Jones, D.A., Narici, M., Gapeyeva, H., Pääsuke, M., Seppet, E., Voit, T., Barnouin, Y., Hogrel, J.Y., Butler-Browne, G. y Maier, A.B. (2013). Diagnostic criteria for sarcopenia and physical performance. Osteoporos Int, 24(10), 681-691.

Boulton, E., Horne M. y Todd, C. (2018). Multiple influences on participating in physical activity in older age: Developing a social ecological approach. Health Expectations, 21(1), 239-248.

Büthe, T. y Jacobs, A. (2015). Qualitative \& multi-method research. Spring, 13(1): 1-7.

Calañas, A. (2005). Alimentación saludable basada en la evidencia. Endocrinología y Nutrición, 52(s2), 8-24.

Campbell, M.J., McComas, A.J. y Petito, F. (1973). Physiological changes in ageing muscles. J Neurol Neurosurg Psychiatry, 36(2), 174-82.

Comercio, (2020). El estrés es el principal motivo de teleasistencia psicológica en Ecuador. Consultado el 2 de mayo de 2020. Disponible en: https://www.elcomercio.com/actualidad/ 
estres-teleasistencia-psicologica-ecuador-pandemia.html.

Comité de Operaciones de Emergencia Nacional, (2020). Informe de Situación COVID-19 Ecuador. Republica del Ecuador. Disponible en: https://www.gestionderiesgos.gob.ec/ wp-content/uploads/2020/04/Informe-de-Situaci\%C3\%B3n-No030-Casos-CoronavirusEcuador-08042020.pdf

Donna, M., Begoña Errasti, I., Gail Low, R., O’Reill, P., Fiona Murphy, R. y Fahy, A. (2020). Identifying contemporary early retirement factors and strategies to encourage and enable longer working lives: A scoping review. Int J Older People Nurs, O(e12313). DOI: https://doi. org/10.1111/opn.12313

Elia, M. (2000). British Association for Parenteral and Enteral Nutrition, Advisory Group on Malnutrition. Guidelines for detection and management of malnutrition. Maidenhead (United Kingdom): British Association for Parenteral and Enteral Nutrition.

Given, L. (2008). The SAGE encyclopedia of qualitative research methods. Thousand Oaks, CA: SAGE Publications.

Grühn, D., Sharifian, N. y Chu, Q. (2016). The limits of a limited future time perspective in explaining age differences in emotional functioning. Psychology and Aging, 31(6), 583-593.

Guerrero, S. (2020). Coronavirus en ecuador: una opinión desde la Academia. La Granja, Revista de Ciencias de la Vida 32(2). Doi: http://doi.org/10.17163/lgr.n32.2020.10.

Gutiérrez, A., Cruz, A. y Saldívar, E. (2020). Gestión de seguridad psicológica del personal sanitario en situaciones de emergencia por COVID-19 en el contexto hospitalario o de aislamiento, Revisión, 3704, 1-20.

Hernández, R., Fernández, C. y Baptista, P. (2014). Metodología de la investigación. México: Trillas. Jancey, J. M., Clarke, A., Howat, P., Maycock, B., Y Lee, A. H. (2009). Perceptions of physical activity by older adults: A qualitative study. Health Education Journal, 68(3), 196-206. Doi: https:// doi.org/10.1177/0017896909339531

Jang, M., Kim, H. y Oh, B. (2018). Comparison of hand-grip strength between normal Korean adults and those with type 2 diabetes: 2014-2015 Korea National. Health and Nutrition Examination Survey. Korean J Fam Pract, 8, 654-661.

Jeong, H., Yim H. \& Song, Y. (2016). Mental health status of people isolated due to Middle East respiratory syndrome. Epidemiol Health, 38, e2016048.

Jensen, G.L., Mirtallo, J., Compher, C., Dhaliwal, R., Forbes, A., Grijalba, R.F., Hardy, G., Kondrup, J., Labadarios, D. y Nyulasi, I. (2010). Adult starvation and disease-related malnutrition: a proposal for etiology-based diagnosis in the clinical practice setting from the International Consensus Guideline Committee. JPEN J Parenter Enteral Nutr, 34, 156-9.

Jin, Y., Cai, L. y Cheng, Z. (2020). A rapid advice guideline for the diagnosis and treatment of 2019 novel coronavirus (2019-nCoV) infected pneumonia (standard version). Military Med Res, 7(4), 1-23. DOI: https://doi.org/10.1186/s40779-020-0233-6

Jones, C.J., Rikli, R.E. y Beam, W.C. (1999). A 30-s chair-stand test as a measure of lower body strength in community-residing older adults. Res Quart Exerc Sport, 70, 113-119. DOI: 10.1080/02701367.1999.10608028.

Khoddam, H., Eshkevarlaji, S., Nomali, M., Modanloo, M., \& Keshtkar, A. (2019). Prevalence of Malnutrition Among Elderly People in Iran: Protocol for a Systematic Review and MetaAnalysis. JMIR research protocols. 8(11), e15334. DOI: https://doi.org/10.2196/15334 
Koman, T. (2020). Why It's So Important You Continue To Hydrate Right Now. Delish. Disponible en: https://www.delish.com/food/a31745872/hydrate-during-coronavirus-quarantine/

Latham, N. y Liu, C. (2010). Strength training in older adults: the benefits for osteoarthritis. Clin Geriatr Med, 26, 445-59.

Le Couteur, D. y Anderson, R. (2020). COVID-19 Through the Lens of Gerontology. The Journals of Gerontology, Series A(glaa077). Doi: https://doi.org/10.1093/gerona/glaa080.

Ley Orgánica de las Personas Adultas Mayores, Asamblea Nacional de la República del Ecuador. (2019). Suplemento del Registro Oficial No. 484. Asamblea Nacional.

Lexell, J., Taylor, C.C. y Sjöström, M. (1988). What is the cause of the ageing atrophy? Total number, size and proportion of different fiber types studied in whole vastus lateralis muscle from 15- to 83-year-old men. J Neurol Sci, 84(2-3), 275-94.

Liu, X., Kakade, M. \& Fuller, C. (2012). Depression after exposure to stressful events: lessons learned from the severe acute respiratory syndrome epidemic. Compr Psychiatry, 53, 15-23.

Liping, S., Zhikai, H., Qinglong, W., Jie, J., Yue, C., Dan, W., Sun, R., Long, L., Xuefeng, C., Yantao, W. \& Yiping, S. (2020). Quantitative species determination based on real time PCR-Can the results be expressed as weight/weight equivalents?. Food Biotechnology, 34(2), 116-131. DOI: 10.1080/08905436.2020.1743305

Ministerio de Salud Pública, (2020), Actualización de casos de coronavirus en Ecuador. Plataforma Gubernamental de Desarrollo Social. Disponible en: https://www.salud.gob.ec/ actualizacion-de-casos-de-coronavirus-en-ecuador/.

Ministerio de Salud Pública, (2020b). Lineamientos operativos para la intervención de salud mental en la emergencia sanitaria. Republica del Ecuador. Disponible en: https://www.salud.gob. ec/wp-content/uploads/2020/07/Lineamientos-operativos-Salud-Mental-Covid-19_2020. pdf

Moynihan, A.B., Van Tilburg, W.A., Igou, E.R., Wisman, A., Donnelly, A.E. y Mulcaire, J.B. (2015). Eaten up by boredom: consuming food to escape awareness of the bored self. Front Psychol, 6(369). DOI: 10.3389 / fpsyg.2015.00369

Myers, N., Bateman, A., Lee, S. \& Silverman, S. (2020). Measurement in Physical Education and Exercise Science (MPEES): A Brief Report on 2019. Measurement in Physical Education and Exercise Science, 24(2), 93-102. DOI: 10.1080/1091367X.2020.1739690

Organización de las Naciones Unidas, (2020), Plan de Respuesta Humanitaria Covid-19 Ecuador. Disponible en: https://reliefweb.int/sites/reliefweb.int/files/resources/20200430-EHPECUADOR-COVID-19.pdf.

Organización mundial de la salud, OMS (2015). Informe mundial sobre el envejecimiento y la salud. Biblioteca de la OMS. ISBN 9789243565040. Disponible en: https://apps.who.int/ iris/bitstream/handle/10665/186466/9789240694873_spa.pdf?sequence $=1$

Osama, T., Pankhania, B., \& Majeed, A. (2020). Protecting older people from COVID-19: should the United Kingdom start at age 60 ? Journal of the Royal Society of Medicine, 1(19). https://doi. org/10.1177/0141076820921107

Organización mundial de la salud, OMS. (2010). Global Recommendations on PA for Health. Disponible en: http://www.who.int/dietphysicalactivity/factsheet_olderadults/en/index. html. 
Organización mundial de la salud, OMS (2020) Water, sanitation, hygiene and waste management for COVID-19. Department of Communications, WHO/2019-nCoV/IPC_WASH/2020.2; 2020. Disponible en: https://apps.who.int/iris/rest/bitstreams/1272446/retrieve

Otzen, T. \& Manterola C. (2017). Técnicas de muestreo sobre una población a estudio. Int. J. Morphol, 35(1), 227-232.

Petretto, D. y Pili, R. (2020). Ageing and COVID-19: What is the Role for Elderly People?. Geriatrics, 5(25). Doi: https://doi.org/10.3390/geriatrics5020025

Piasecki, M., Ireland, A., Jones, D.A. y McPhee, J.S. (2016). Age-dependent motor unit remodelling in human limb muscles. Biogerontology, 17(3), 485-96.

Power, S.E., Jeffery, I.B., Ross, R.P., Stanton, C., O'Toole, P.W., O'Connor, E.M. y Fitzgerald, G.F. (2014), Food and nutrient intake of Irish community-dwelling elderly subjects: who is at nutritional risk?. J Nutr Health Aging, 18(6), 561-72.

Ready, R., Åkerstedt, A. \& Mroczek, D. (2011). Emotional complexity and emotional well-being in older adults: Risks of high neuroticism. Aging \& Mental Health, iFirst, 1-10. DOI: 10.1080/13607863.2011.602961.

Rodríguez-Martín B. y Meule, (2015). A. Food craving: new contributions on its assessment, moderators, and consequences. Frontiers in Psychology, 6(21), 1-3. DOI: 10.3389/ fpsyg.2015.00021.

Samuelsson, J., Rothenberg, E., Lissner, L., Eiben, G., Zettergren, A., \& Skoog, I. (2019). Time trends in nutrient intake and dietary patterns among five birth cohorts of 70-year-olds examined 1971-2016: results from the Gothenburg H70 birth cohort studies, Sweden. Nutrition journal, 18(1), 66. DOI: https://doi.org/10.1186/s12937-019-0493-8.

Scott, J. (2006). Documentary research. Thousand Oaks, CA: Sage Publications.

Seong, J., Ahn, H., Park, Y., Shin, S. y Hyuk H. (2020). Association between Aerobic Exercise and Handgrip Strength in Adults: A Cross-Sectional Study Based on Data from the Korean National Health and Nutrition Examination Survey (2014-2017). J Nutr Health Aging, 2. DOI: https://doi.org/10.1007/s12603-020-1372-x

Shakouri1, N. (2014). Qualitative Research: Incredulity toward Metanarrativeness. Journal of Education and Human Development, 3(4), 671-680.

Shlisky, J., Bloom, D., Beaudreault, A., Tucker, K., Keller, H., Freund, Y., Fielding, R., Cheng, F., Jensen, G., Wu, D. \& Meydani, S. (2017). Nutritional Considerations for Healthy Aging and Reduction in Age-Related Chronic Disease. Advances in nutrition, 8(1), 17-26. Doi: https:// doi.org/10.3945/an.116.013474

Taylor, D. (2014). Physical activity is medicine for older adults. Postgraduate Medical Journal, 90, 26-32.

Thurnham, D.I. (1997). Micronutrientes y función inmune: algunos desarrollos recientes. J Clin Pathol, 50, 887-91.

Volkwein, K. y McConatha, J. (2018). Physical Activity and Health. London: Routledge.

WaldroupH, P. y Hellwig, H. (2000). The Potential Value of Morning and Afternoon Feeds for Laying Hens1. The Journal of Applied Poultry Research, 9(1), 98-110.

Wang, Y. \& Chen, A. (2019). Two Pathways Underlying the Effects of Physical Education on Out-Of-School Physical Activity. Research Quarterly for Exercise and Sport. DOI: 10.1080/02701367.2019.1656325 
Wu, C., Chen, X., Cai. Y., Xia, J., Zhou, X. y Xu, S. (2020). Risk factors associated with acute respiratory distress syndrome and death in patients with coronavirus disease 2019 pneumonia in Wuhan, China. JAMA Intern Med, 20. 58-67. DOI: 10.1001/jamainternmed.2020.0994.

Yılmaz, C. y Gökmen, V. (2020). Neuroactive compounds in foods: Occurrence, mechanism and potential health effects. Food Research International, 128(108744). DOI: https://doi. org/10.1016/j.foodres.2019.108744

Zaragoza-Martí, A., Ruiz-Robledillo, N., Sánchez-SanSegundo, M., Albaladejo-Blázquez, N., Hurtado-Sánchez, J. A. \& Ferrer-Cascales, R. (2020). Eating Habits in Older Adults: Compliance with the Recommended Daily Intakes and Its Relationship with Sociodemographic Characteristics, Clinical Conditions, and Lifestyles. Nutrients, 12(2), 446. DOI: https://doi. org/10.3390/nu12020446 